\title{
On the design, feasibility, and implementation of a Bluetooth MANET-based routing application
}

\author{
Ryohei Saka (i) ${ }^{\text {a) }}$, Temma Ohtani ${ }^{\text {b) }}$, Kazuki Fujita ${ }^{\text {c) }}$, Eitaro Kohno ${ }^{d)}$, \\ and Yoshiaki Kakuda ${ }^{\mathrm{e})}$ \\ Graduate School of Information Sciences, Hiroshima City University, \\ 3-4-1 Ozuka-Higashi, Asaminami-ku, Hiroshima 731-3194, Japan \\ a)saka@nsw.info.hiroshima-cu.ac.jp \\ b)ohtani@nsw.info.hiroshima-cu.ac.jp \\ c)fujita@nsw.info.hiroshima-cu.ac.jp \\ d)kouno@hiroshima-cu.ac.jp \\ e)kakuda@hiroshima-cu.ac.jp
}

\begin{abstract}
Bluetooth MANETs, which are wireless networks with Bluetooth-enabled terminals, only have proposed flooding-based methods for one-to-many communication among terminals. In general, however, to develop and implement useful applications, we have to prepare routingenabled frameworks for Bluetooth MANETs. In this paper, we have proposed a new Bluetooth MANET-based routing scheme through the development of an attendance confirmation system. We also have implemented the system on Android- and Raspberry Pi-based terminals, and have conducted 10 terminals-based experiments. As a result, we have confirmed that our proposed method worked properly.
\end{abstract}

Keywords: Bluetooth, Bluetooth MANETs with routing, many-to-one communication, attendance confirmation

Classification: Network

\section{References}

[1] Y. Minami, R. Saka, E. Kohno, and Y. Kakuda, "Data forwarding schemes to adapt connectivity among terminals for Bluetooth MANET with delay and disruption tolerance," IEICE Trans. Commun. (Japanese Edition), vol. J102-B, no. 5, pp. 356-365, May 2019. DOI:10.14923/transcomj.2018NSP0004

[2] Y. Minami, R. Saka, E. Kohno, and Y. Kakuda, "A fast connection establishment method using classic Bluetooth and Bluetooth low energy for Bluetooth MANET," IEICE Trans. Commun. (Japanese Edition), vol. J102-B, no. 8, pp. 545-554, Aug. 2019. DOI:10.14923/transcomj.2018WFP0013

[3] T. Ohtani, E. Kohno, and Y. Kakuda, "On relationship between timeout and latency of connection re-establishment for control packetloss scenario in Bluetooth MANETs," Proc. 2018 Sixth International Symposium on Computing and Networking (CANDARW), 11th International Workshop on Autonomous Self-Organizing Networks (ASON), pp. 42-46, Nov. 2018. DOI:10. 1109/CANDARW.2018.00016 
[4] Y. Minami, N. Kajikawa, R. Saka, Y. Nakao, E. Kohno, and Y. Kakuda, "Arbitration-based deadlock mitigation mechanism for fast connection establishment in autonomous self-organized Bluetooth MANETs," 2018 IEEE SmartWorld/SCALCOM/UIC/ATC/CBDCom/IOP/SCI, at ADSN2018, pp. 1611-1616, Oct. 2018. DOI:10.1109/SmartWorld.2018.00276

[5] N. Kajikawa, Y. Minami, E. Kohno, and Y. Kakuda, "On availability and energy consumption of the fast connection establishment method by using Bluetooth classic and Bluetooth low energy," Proc. Fourth International Symposium on Computing and Networking (CANDAR 2016), 9th International Workshop on Autonomous Self-Organizing Networks (ASON 2016), pp. 286-290, Nov. 2016. DOI:10.1109/CANDAR.2016.0058

[6] C. M. Yu and E. L. Lin, "Reliable formation protocol for Bluetooth hybrid single-hop and multi-hop networks," IEEE Netw., vol. 32, no. 2, pp. 120-125, Oct. 2018. DOI:10.1109/MNET.2017.1700008

[7] C. Bisdikian, "An overview of the Bluetooth wireless technology," IEEE Commun. Mag., vol. 39, no. 12, pp. 86-94, Dec. 2001. DOI:10.1109/35. 968817

[8] Bluetooth SIG, "Specification of the Bluetooth system - covered core package version: 2.1+EDR -," July 2007. https://www.bluetooth.org/docman/handlers/ downloaddoc.ashx?doc_id=456435 (2019-10-03).

[9] Bluetooth SIG, "Specification of the Bluetooth system - covered core package version: 4.2 -," Dec. 2014. https://www.bluetooth.org/docman/handlers/ downloaddoc.ashx?doc_id=441541 (2019-10-03).

[10] Y. Minami, Y. Kitaura, Y. Tsutsui, E. Kohno, S. Inoue, T. Ohta, and Y. Kakuda, "Implementation and evaluation of dual-purpose normal and disaster situations system based on delay and disruption tolerant Bluetooth MANETs," IJCNS, vol. 8, no. 9, pp. 342-357, Sept. 2015. DOI:10.4236/ijcns.2015.89033

[11] M. Nishi, H. Kozato, Y. Ota, and K. Shin, "Development of disaster monitoring techniques in the grass-root information distribution system for detecting landslide dangers," 2018 IEEE SmartWorld/SCALCOM/UIC/ATC/ CBDCom/IOP/SCI, at ADSN2018, pp. 1599-1604, Oct. 2018. DOI:10. 1109/SmartWorld.2018.00274

\section{Introduction}

Recently, research on Bluetooth MANETs [1, 2, 3, 4, 5], which consist of mobile Bluetooth-enabled terminals $[6,7,8,9]$, have been conducted. Bluetooth MANETs are one prospective communication methodology for a dual-purpose normal and disaster situations system $[10,11]$. So far, Bluetooth MANETs only provided a oneto-many communication method, which corresponds to flooding in MANETs. In order to enhance the applicability of the system, we have to propose and develop many-to-one or other communication methodologies. In this paper, we propose a new many-to-one communication method for Bluetooth MANETs. We also implement our proposed method and confirm its correctness.

\section{Proposed method (Bluetooth MANETs with routing)}

\subsection{Overview}

As we mentioned in Section 1, Bluetooth MANETs have so far only provided a one-to-many communication method. In our proposed method, we have proposed a 


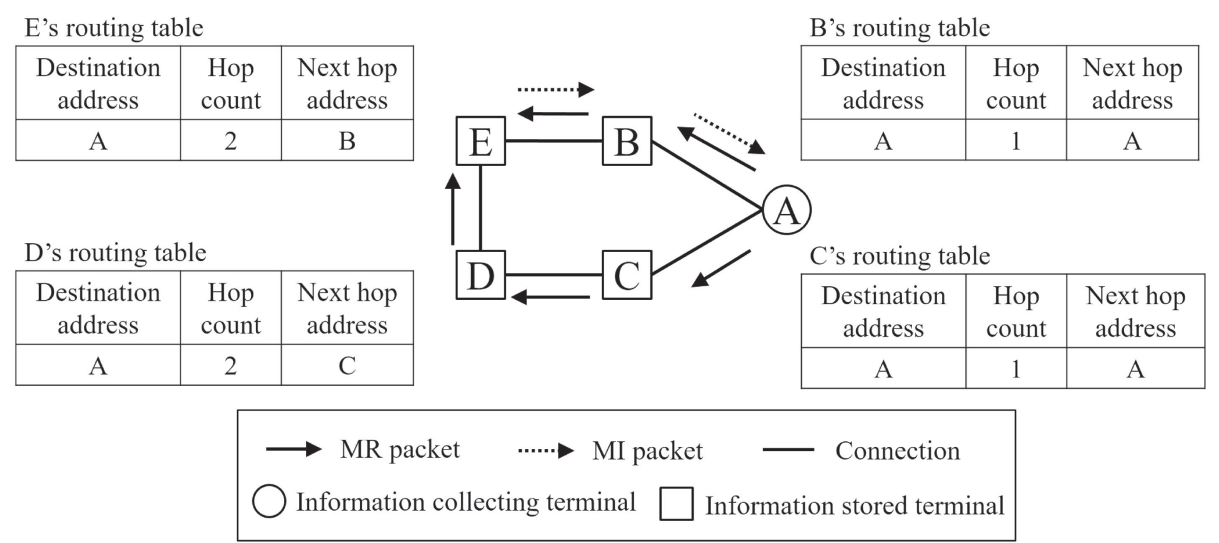

Fig. 1. Example of routing procedures of our proposed method

new many-to-one communication method with a routing function for Bluetooth MANETs. In order to realize the routing function, our proposed method utilizes a flooding-based one-to-many communication method. In this paper, we define a terminal that collects information and a terminal that sends information are an "Information collecting terminal," and an "Information stored terminal," respectively.

\subsection{Routing}

First, an information collecting terminal broadcasts a member request (MR) packet to adjacent terminals. The MR packet contains the source (information collecting) terminal's address and the hop count from the source terminal. The adjacent (intermediate) terminals re-broadcast the received MR packets in a "flooding" manner. When an information stored terminal receives the MR packet, it registers or updates its routing table then sends back its stored data. Fig. 1 shows the routing procedures of our proposed method.

\subsubsection{The procedures of registration and update of the routing table}

The routing table contains the destination address, hop counts, and the next hop address as an entry. When a terminal receives an MR packet, it searches its own routing table as to whether the routing table has the entry for the destination address that is included in the received MR packet. When the routing table does not have the entry for the destination address, the terminal creates and registers the new entry for the destination address in the routing table. Otherwise, the terminal compares the hop counts between the received MR packet and the entry of the routing table. When the hop count in the received MR packet is smaller than that in the routing table, the terminal updates the hop counts and the next hop address of the entry in the routing table by using the hop count value and the next hop address in the MR packet.

Suppose the terminals, as shown in Fig. 1, already have established connections among them. When terminal A broadcasts an MR packet and adjacent terminals $\mathrm{B}$ and $\mathrm{C}$ receive it, the routing tables in terminals $\mathrm{B}$ and $\mathrm{C}$ have no entries for the destination address of terminal $\mathrm{A}$. Terminals $\mathrm{B}$ and $\mathrm{C}$ create new entries for the destination address by referring to their received MR packets. Then, in their new MR packets, terminals B and C increment the hop counts by one before 
broadcasting the packets. When terminals $\mathrm{D}$ and $\mathrm{E}$, which are adjacent to terminals $B$ and $C$, receive MR packets, they create and register their new entries in the routing tables. When terminal D broadcasts and terminal E receives terminal D's MR packet, terminal E compares the hop counts between the received MR packets and the routing table of terminal E. Since the routing table of terminal E already has a smaller hop count entry, the entry does not update.

\subsubsection{The procedures of data packets by using the routing table}

The information stored terminals that received MR packets send back what we call "member information (MI) packets" which contain the destination address and the stored information to. When an information stored terminal receives MI packets from other terminals, it also forwards the MI packets by referring to its routing table.

When terminal E, as shown in Fig. 1, sends back its MI packet, it searches terminal A's entry in the routing table and forwards its MI packet to terminal B. Then terminal A, the information collecting terminal, can collect terminal E's stored information successfully.

\section{Real world application: Bluetooth-MANET-based student attend- ance confirmation system}

In this paper, we also have developed and implemented a system to confirm student attendance using the routing method for Bluetooth MANETs in order to confirm the feasibility of our proposed routing method. The system consists of an information collecting terminal, which is for an attendance information collector(s) such as a teacher of a lecture, and multiple information stored terminals, which are for attendance information providers such as students. The information stored terminals have preliminarily distributed unique information such as student IDs.

The following is the procedure of our proposed system: first, the information collecting terminal broadcasts the MR packet to start to collect the attendance information. Each information stored terminal performs flooding to forward its received packets and construct the routing table as described in section 2.2.1. Then, each information stored terminal sends back the MI packet to the information collecting terminal. When an information stored terminal receives other information stored terminals' MI packets, the terminal refers to its routing table to forward the MI packet(s). With this system, a classroom can take fast and reliable attendance data.

\section{Experiments}

\subsection{Overview}

We have implemented our proposed student attendance confirmation system to Android- and Raspberry Pi-based terminals to confirm the latency of the system.

\subsection{Experimental configuration}

In the experiments, we prepared one SONY XPERIA Z3+ (Android OS ver. 7.1.1, and Bluetooth ver. 4.1) terminal for the information collecting terminal, and 10 Raspberry Pi3 model B (Raspbian ver. 9.1, and Bluetooth ver. 4.1) terminals for 


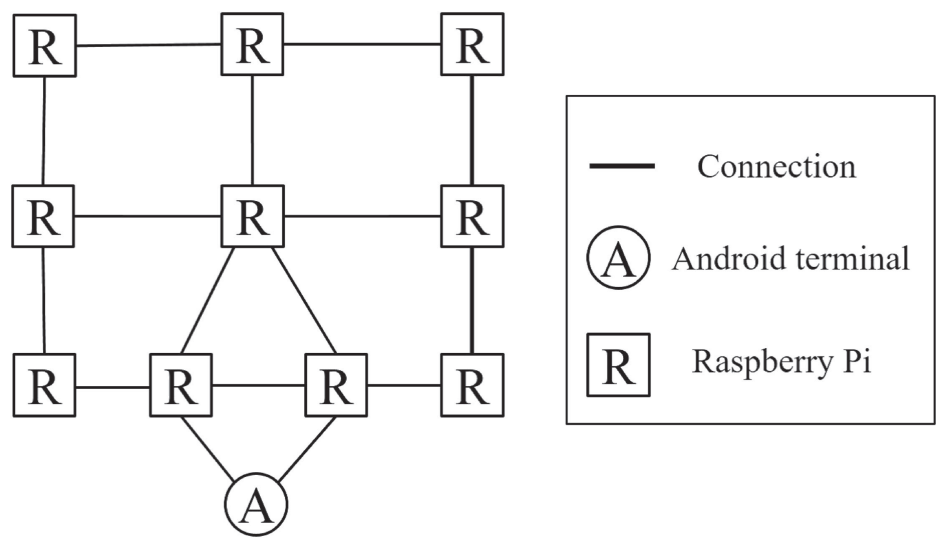

Fig. 2. Network topology

the information stored terminals. In these experiments, terminals were not moved. We also preliminarily established Bluetooth connections as shown in Fig. 2 to confirm the effectiveness when our proposed system must use multi-hop data packet forwarding in Bluetooth MANETs. The measurement field is a lecture room that can hold 320 people at Hiroshima City University. The field is also equipped with IEEE $802.11 \mathrm{~g} / \mathrm{n} / \mathrm{h}$ Wi-Fi, which uses the same frequency band and could be a source of interference. In these experiments, four of the authors participated.

\subsection{Evaluation items}

In the experiments, we have gauged the collection latency of all information stored terminals. We define the collection latency as the waiting time between when the Android terminals start to send MR packets and when the Android terminal can collect MI packets from all information stored terminals.

In addition, we have constructed network topology as shown in Fig. 2 in order to observe the behavior of our proposed method.

\subsection{Results}

Fig. 3 shows the experimental results. We have conducted the 30 trials. The horizontal and vertical axes show each trial number and the collection latency,

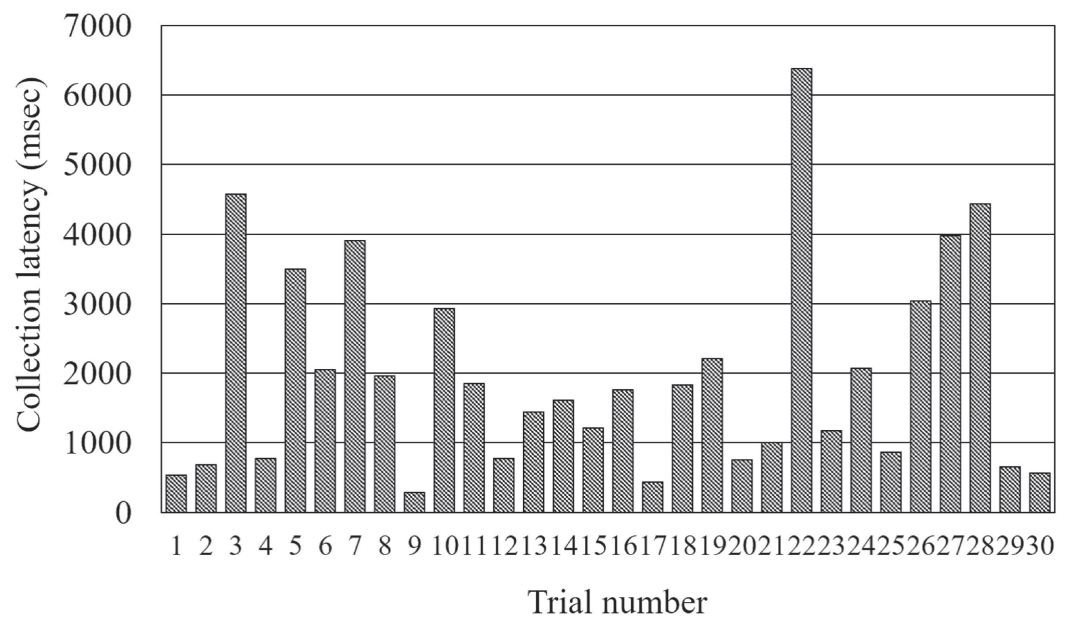

Fig. 3. Collection latency 
respectively. The maximum and the minimum latencies were 6329 (ms) and 290 (ms), respectively. In addition, the average latency was 1975.6 (ms) and the 95 percent confidence interval was 550.9. From the results, we could confirm that our proposed system and our proposed method could work properly in the 10 terminal's environments.

\section{Conclusion}

In this paper, we have proposed a new routing method for Bluetooth MANETs and have implemented it in the student attendance confirmation system to confirm our proposed method. We have also conducted experiments on the latency of the system in 10 terminal-wide networks. We have found that the system could collect 10 terminals' attendance information within 7 seconds even in Wi-Fi enabled environments.

While we developed the normal situation-oriented attendance confirmation system for lectures in university, the system can be applied to the prospective disaster-oriented attendance system for evacuees in a place of refuge.

For future works, we are planning to conduct experiments in large-scale networks.

\section{Acknowledgments}

This research is supported by the Ministry of Internal Affairs and Communications, Japan, Strategic Information and Communications R\&D Promotion Programme (SCOPE), JSPS KAKENHI Grant Number (C) (No. 17K00130, and 17K00131). 